\title{
The Healing Effect of Aloe Vera Gel on Acetic Acid-Induced Ulcerative Colitis in Rat
}

Negar Hassanshahi ${ }^{1}$, Seyed Jalil Masoumi ${ }^{2,3,{ }^{*}}$, Davood Mehrabani ${ }^{4}$, Seyedeh Sara Hashemi ${ }^{5}$, Morteza Zare ${ }^{2}$

1. Student Research Committee, School of Nutrition and Food Sciences, Shiraz University of Medical Sciences, Shiraz, Iran

2. Nutrition Research Center, School of Nutrition and Food Sciences, Shiraz University of Medical Sciences, Shiraz, Iran

3. Gastroenterohepatology Research Center, Shiraz University of Medical Sciences, Shiraz, Iran

4. Stem Cell and Transgenic Technology Research Center, Shiraz University of Medical Sciences, Shiraz, Iran

5. Burn and Wound Healing Research Center, Shiraz University of Medical Sciences, Shiraz, Iran

\footnotetext{
* Corresponding Author:
}

Seyed Jalil Masoumi, MD, Mph, PhD Nutrition Research Center, Department of Clinical Nutrition, School of Nutrition and Food Sciences, Shiraz University of Medical Sciences, Shiraz, Iran Telefax: + 987132332313 Email: masoumi7415@gmail.com, massoumij@sums.ac.ir

Received: 27 Dec. 2019

Accepted: 10 May. 2020

\section{ABSTRACT}

\section{BACKGROUND}

The use of herbal and synthetic compounds can be effective in improving the areas and repair of tissues that have been affected during the processes like what happens in ulcerative colitis (UC) as a common inflammatory disorder. According to the beneficial effects of aloe vera, in this study, we aimed to assess the therapeutic effects of oral aloe vera gel on acetic acid-induced colitis in rats by histopathological and molecular analysis of Bax, and BCL-2 genes expression (using RT-PCR technique) in colon tissue samples.

\section{METHODS}

This experimental study comprised 32 adult male Sprague Dawley rats weighting $220 \pm 20 \mathrm{~g}$ that were randomly divided into four groups as follows. The control group (healthy rats), colitis group in which UC was induced by transrectal administration of $3 \%$ acetic acid with no treatment, oral form of sulfasalazine group in which UC was induced by transrectal administration of $3 \%$ acetic acid, then was treated by oral administration of sulfasalazine $500 \mathrm{mg} / \mathrm{kg}$ body weight, and the fourth group which received oral form of aloe vera gel $(200 \mathrm{mg} / \mathrm{kg})$ for 21 days, respectively after induction of UC. Then, the therapeutic effects of treatment groups were compared with the control group and the colitis group with no treatment, by the assessment of histopathological and molecular changes in the colon tissues of rats on the $7^{\text {th }}, 14^{\text {th }}$, and $21^{\text {st }}$ days. Finally, the collected data were analyzed using statistical tests.

\section{RESULTS}

Histologically, aloe vera gel treatment could reduce and heal colon tissue damages in induced colitis. Also, this gel reduced apoptosis in rat's colon with acetic acid-induced colitis, which showed in significantly decreased in Bax mRNA expression and significantly increased BCL-2 mRNA expression compared with the colitis group with no treatment.

\section{CONCLUSION}

Aloe vera gel has a significant effect on the treatment of UC in rat because of the beneficial effect that was found from aloe vera such as decreasing the severity of colitis as evidenced by histopathological findings, and with respect to apoptosis and gene expression that were related to wound healing process, and suppression of the elevation of Bax mRNA with the upregulation of $\mathrm{Bcl}-2$, which can be considered effective in the treatment of UC.

\section{KEYWORDS:}

Inflammatory bowel disease, Aloe vera gel, Male rat, Ulcerative colitis, Bax, BCL-2 gene expression.

\section{Please cite this paper as:}

Hassanshahi N, Masoumi SJ, Mehrabani D, Hashemi SS, Zare M. The Healing Effect of Aloe Vera Gel on Acetic Acid-Induced Ulcerative Colitis in Rat. Middle East J Dig Dis 2020;12: 154-161. doi: 10.34172/mejdd.2020.177.

\section{INTRODUCTION}

Ulcerative colitis (UC) is a chronic, relapsing inflammatory disorder of the gastrointestinal system, which can induce inflammation and damages in mucosal layers of the colon, especially the rectum. ${ }^{1} \mathrm{UC}$ activity ranges from weak to 
severe and can affect the quality of life of the patient directly. ${ }^{2}$ The most dangerous and fatal side effects of this inflammatory disease are anemia, obstruction of the gastrointestinal tract, and nutritional deficiencies due to the lack of absorption of food in the intestines. On the other hand, risk of cancers such as colorectal and lymphoma cancers were shown to be high in these patients, which can explain the increased mortality rates in the affected patients. ${ }^{3}$ Although the causes and the exact pathogenesis of UC are not yet known, generally, research on UC suggests that the changes in the immune system tolerance of the gastrointestinal tract can be responsible for the pathogenesis, which begins with certain factors and leads to a long-lasting, imbalanced, and severe inflammatory responses in the intestinal tissue of people that are genetically predisposed. , $, 5,6^{-}$

These factors can lead to intestinal epithelial cell damages and apoptosis characterized by a rise in Bax mRNA, and a decrease in BCL-2 mRNA as an anti-apoptotic gene, and finally an increase happens in mitochondrial membrane permeability to cytochrome $\mathrm{c}$ and also results in activation of caspase, which is responsible for apoptosis. ${ }^{\text {? }}$ Since UC is a disease with multiple causes and does not have a definitive treatment method, there are several management methods for UC, including the changes in dietary habits and lifestyle, drug therapy, and in some cases, surgery is necessary. Because the available treatment methods have side effects and cannot cure the disease completely, researchers are always looking for more effective and low-risk treatment methods for this disease. ${ }^{8,9}$

The use of alternative therapies and natural dietary compounds for healing purposes is increasing among the therapists and patients with UC, among them, aloe vera with the scientific name (Aloe vera Linne), is under research. This plant is a cactus-like plant with green and stretched leaves that contains viscous and transparent gel with approximately $99.5 \%$ water and about 75 types of active compounds such as vitamins, minerals, enzymes, hormones, carbohydrates, lumps, proteins, amino acids, sterols, and other organic derivatives. It is native to Asia, Africa, and other tropical regions of the world with several therapeutic effects have been found during various studies on this plant. ${ }^{10,11,12,13}$ Therefore, according to the antioxidant, anticancer, anti-inflammatory, analgesic, antimicrobial, and wound healing effects of aloe vera reported in various studies, aloe vera can be used in healing of UC to provide a protective effect on inflammation in the affected tissue. ${ }^{10,11,12,14,15}$

Diagnosis of inflammatory bowel disease is based on the clinical and endoscopic findings and biopsy of the patient's intestinal mucosa. Laboratory measurements, ultrasound, and radiological examinations can also be useful in assessing and monitoring of the disease activity and in differentiating $\mathrm{UC}$ from other forms of colitis with similar characteristic. In general, the accuracy of diagnosis in UC is based on methods that assess the extent and severity of inflammation. ${ }^{16}$ So this study was undertaken to evaluate the therapeutic healing effect of aloe vera gel in injured tissue by histopathological and molecular methods.

\section{MATERIALS AND METHODS}

\section{Preparation of aloe vera gel}

The fresh, mature leaves of aloe vera were prepared and approved in terms of race and species by the herbarium of the Pharmacy School of Shiraz University of Medical Sciences and then were transferred to the plant laboratory of Comparative and Experimental Medical Center of Shiraz University of Medical Sciences. The leaves were then washed and disinfected with water and alcohol, after its fragmentation and pulp extraction under sterile conditions, and were homogenized by Homogenizer. Then the gel solution was condensed at $60^{\circ} \mathrm{C}$ in the evaporator device for one hour and was dried in the incubator system at $60^{\circ} \mathrm{C}$. Finally, it was powdered and stored at $-21^{\circ} \mathrm{C}$ in the freezer.

\section{Preparation and maintenance of animals:}

32 male Sprague Dawley rats $220 \pm 20 \mathrm{~g}$ were prepared from the central animal house of Shiraz University of Medical Sciences. They were kept in separate cages in a ventilated room of animal house under the standard conditions. They were allowed to have free access to water and food during the experiments except during the fasting time. Animal feedings were done with prepared standard plates. The practical activities of this intervention were conducted in the central animal house of Shiraz University of Medical Sciences and were done according to the laboratory animal ethics protocols. 


\section{Methods}

\section{Induction of ulcerative colitis}

Animals were fasted for 24 hours with free access to water to drain their gut contents. The rats were anesthetized with diethyl ether. Then enema needle was entered up to $8 \mathrm{~cm}$ into the rectum, and transrectal administration of $2 \mathrm{ml}$ of $3 \%$ acetic acid solution was performed. In this stage, the rats were kept in a vertical head-down position to avoid leakage of acetic acid. After 24 hours, the rats had diarrhea or rectal bleeding, which indicated the induction of colitis.

\section{Study Design}

This experimental study comprised four groups of eight rats, each that were randomly divided as follows:

Group 1: Control group with healthy rats.

Group 2: Colitis group in which UC was induced by transrectal administration of acetic acid $3 \%$ with no treatment.

Group 3: Sulfasalazine drug $500 \mathrm{mg} / \mathrm{kg}$ of body weight dose was administered orally for 21 days after induction of colitis by transrectal administration of acetic acid.

Group 4: Aloe vera gel $200 \mathrm{mg} / \mathrm{kg}$ of body weight was administered orally for 21 days after induction of colitis by transrectal administration of acetic acid.

To provide the desired concentration of sulfasalazine and aloe vera gel, which were administered to rats through oral gavage daily, a certain amount of their powder was mixed with a specified volume of distilled water.

\section{Evaluation methods}

Two methods of histopathological and molecular analysis of gene expression were used for the assessment of the colon tissue of rats.

\section{Histopathological assessment}

After the intervention, the rats were killed in the $7^{\text {th }}$, $14^{\text {th }}, 21^{\text {st }}$ day with $\mathrm{CO}_{2}$, in the special chambers and 10 $\mathrm{cm}$ from the end of their colon was removed (biopsy). Then half of the colon tissue samples were fixed for histopathological assessment in formalin-10\%, and in the next stage, the paraffin blocks were prepared from the samples, and then sections of 5-micrometer thickness were prepared and stained with hematoxylin and eosin
(H\&E) method. These processing and assessment of specimens were performed by a histopathologist who was unaware of the treatments. The process was done using a microscope equipped with a camera to take digital images from the colon sections. The images were assessed in terms of histopathological indices like the structure of tissue, the presence of inflammation, ulcer, edema, necrosis, and infiltration of leukocytes into the tissue.

\section{Molecular analysis of gene expression (RT-PCR technique)}

The other half of the colon tissue specimens were stored in a nitrogen tank for molecular evaluations. To assess the expression of inflammation and apoptosisassociated target genes (Bax, BCL-2) expression in colon tissue samples were removed from the nitrogen tank, and after colon tissue homogenization, total RNA was extracted from them by using RNA extraction kit. Then by using random primers and cDNA synthesis kit, their cDNA were made, and then real-time PCR technique was performed by using the specific primers of Bax, and Bcl-2 genes (Table 1). Finally, the frequency of gene expression ratio in different samples was measured using a $\Delta \triangle \mathrm{CT}$ method, and according to the manufacturer's instructions, the results were expressed with a diagram based on the fold change numbers of mRNA expression of Bax, and BCL-2 apoptotic genes activity in the rats' colon. Parametric data were expressed as mean \pm SEM, and the differences between the groups, by the normal distribution, were done using one-way ANOVA. Repeated measures ANOVA analysis was applied to compare the process of changing variables under investigation. Statistical analysis was performed using Graph Pad Prism 6 software. The significance level was defined as $p<0.05$.

\section{RESULTS}

\section{Histopathological assessment}

In order to investigate whether oral aloe vera gel has healing effects on histopathological damage in the colon of rats with acetic acid-induced colitis, microscopic slide sections of the colon samples were assessed for histopathological indices such as the structure of tissue, the presence of inflammation, ulcer, edema, necrosis, and infiltration of leukocytes into the tissue. These following 
Table 1: Primer sequences used for real-time PCR

\begin{tabular}{lcc}
\hline mRNA species & Primer sequence & Primer length \\
\hline Ra BAX $\mathrm{F}$ & CTGCAGAGGATGATTGCTGA & $174 \mathrm{bp}$ \\
\hline Ra BAX R & GATCAGCTCGGGCACTTTAG & $134 \mathrm{bp}$ \\
\hline Ra Bcl2 F & ATCGCTCTGTGGATGACTGAGTAC & \\
\hline Ra Bcl2 R & AGAGACAGCCAGGAGAAATCAAAC & $244 \mathrm{bp}$ \\
\hline R-B2m -F & CGTGCTTGCCATTCAGAAA & \\
\hline R-B2m $-\mathrm{R}$ & ATATACATCGGTCTCGGTGG & \\
\hline
\end{tabular}

pictures were taken to evaluate the results from the slides of colon tissue sections in different groups with different magnification (Figure1). Histopathological results of colon tissue samples of rats in different groups indicated: In the control group normal structure was shown with no symptoms of UC, while in the colitis group with no treatment, most of the symptoms of UC histopathology were observed. Sulfasalazine therapy could not improve the severity of symptoms of UC histopathology, while aloe vera gel treatment could reduce and heal colon tissue damages in induced colitis by reducing the severity of symptoms of UC shown on histopathologic evaluation.

\section{Molecular assessment}

In the pathogenesis of UC, increase in production and secretion of inflammatory mediators and reactive oxygen species, and infiltration of leukocytes, eventually lead to inflammation, ulcer, bleeding, intestinal damage, diarrhea, and induction of apoptosis. In this study, in order to investigate whether aloe vera gel can reduce apoptosis in rat's colon with acetic acid-induced colitis, the mRNA expression of apoptotic genes was assessed. If this gel is effective, the expression of pro-apoptotic, Bax mRNA decreases, and increases in anti-apoptotic BCL-2 mRNA expression. Bax mRNA expression is shown in chart 1 . In chart 2 the expression of the Bcl-2 mRNA of the prepared samples is shown in different groups, on the $7^{\text {th }}, 14^{\text {th }}$, and $21^{\text {st }}$ days. In the charts, the statistically significant difference in groups with the control group are shown with * sign. $p$ value $<0.05$ are shown with $*$ and $p$ value $<0.01$, are shown with $* *$ sign. And also statistically significant difference in the colitis group with no treatment is shown with \# sign. $p$ value $<0.05$ are shown with \# and $p$ value $<0.01$, are shown with \# \# sign.

Molecular assessments revealed that treatment with sulfasalazine led to a significant increase in Bax mRNA expression compared with the control group over the 7-, 14-, and 21-day periods (the greatest change was in the $14^{\text {th }}$ day), but showed a significant decrease in Bax mRNA expression compared with the colitis group with no treatment in the $21^{\text {st }}$ day. This drug also led to a significant decrease in BCL-2 mRNA expression compared with the control group over the 7-, 14-, and 21-day periods but showed a significant increase in BCL-2 mRNA expression compared with the colitis group with no treatment in the $21^{\text {st }}$ day.

Molecular assessments revealed that treatment with aloe vera gel resulted in a significant decrease in Bax mRNA expression compared with the colitis group with no treatment, over the 7-, 14-, and 21-day periods, and resulted in a significant increase in BCL-2 mRNA expression compared with the colitis group with no treatment in the $21^{\text {st }}$ day. In this study, aloe vera gel showed the greatest changes in decreasing apoptosis induction on the $21^{\text {st }}$ day after treatment.

\section{DISCUSSION}

In UC, the impaired immune response of the intestinal mucosa against environmental factors and pathogens leads to an increase in production and secretion of inflammatory mediators, which results in inflammation, damages in intestinal cells, pain, bleeding, diarrhea, and induction of apoptosis. ${ }^{16,17}$ To induce experimental UC, different methods such as administration of trinitrobenzene sulphonic acid (TNBS), ${ }^{17}$ dextran sulfate sodium, and acetic acid have been used. ${ }^{18}$ Induction of colitis with acetic acid is one of the standard experimental models for creation of UC, which is similar in many histopathological features to UC in humans including infiltration of neutrophils, acute inflammation, injuries in mucosal and submucosal layers, and necrosis in colon tissue. ${ }^{19}$

Given that nutrition and diet can affect the patients 

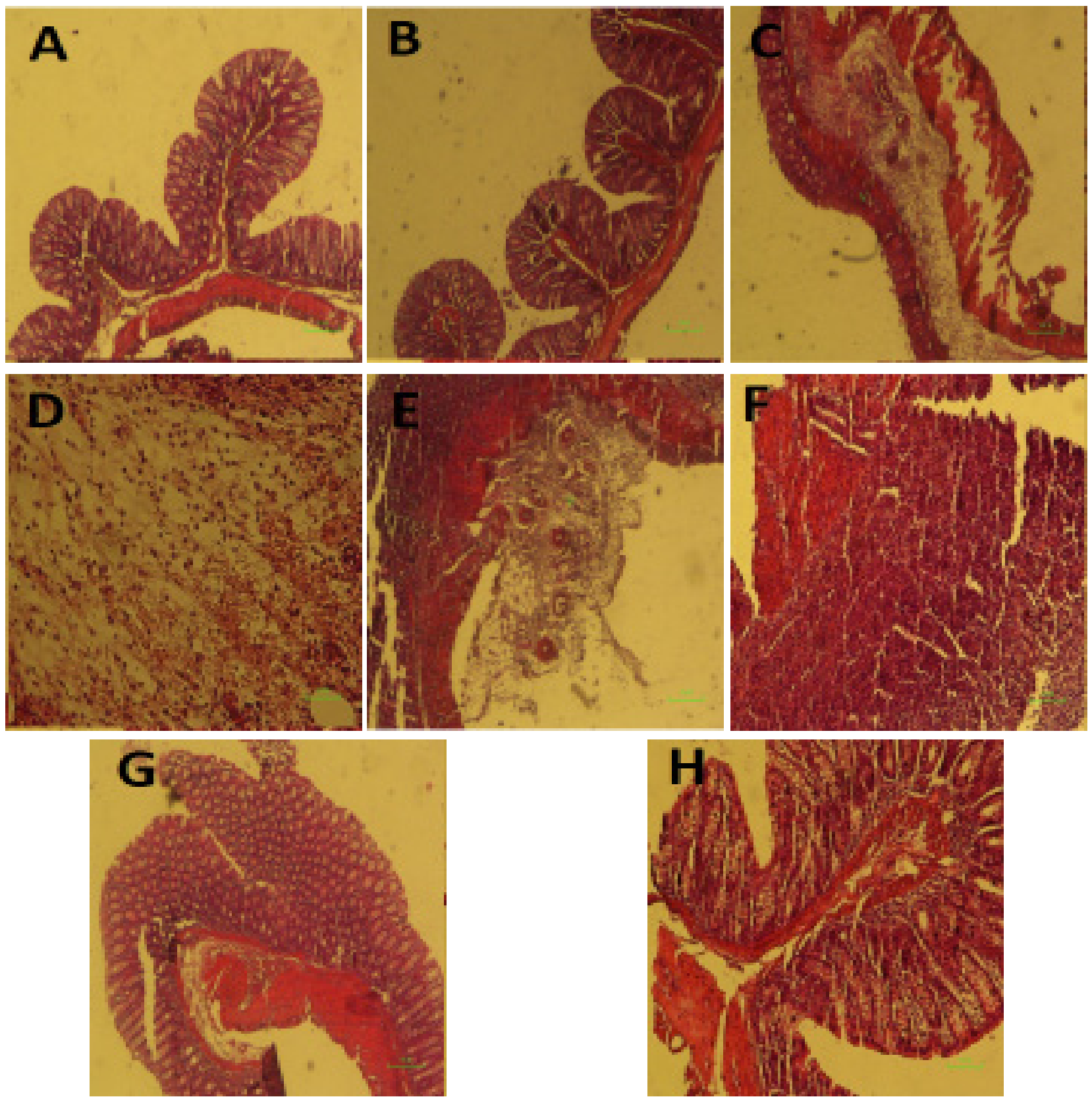

Fig.1: Representative of the microscopic slide sections of the colon samples in rats: (A) Normal colon sample magnification (x 40), showed the normal structure of mucosa with an intact epithelial surface, submucosa, and muscular layer. (B) Normal colon sample, magnification (x 100), showed the normal structure of layers. (C) Acetic acid-induced colitis with no treatment, magnification (x 40), showed an inflammatory reaction, presence of ulcer, inflammation, edema, and diffuse infiltration of leukocytes in the submucosal layer. (D) Acetic acid-induced colitis with no treatment, magnification (x 200), showed inflammation in the submucosal layer with ulceration and edema. (E) Acetic acid-induced colitis+oral administration of sulfasalazine, magnification (x 40), revealed inflammatory reaction in the serosa layer. (F) Acetic acid-induced colitis + oral administration of sulfasalazine sample, magnification (x 100) revealed inflammatory reaction with the dominance of lymphocyte and fewer neutrophils with tissue necrosis. $(\mathrm{G})$, (H) Acetic acidinduced colitis+oral administration of aloe vera gel, magnification (x 40), and (x 100), revealed the improvement of inflammation and the normal tissue.

with inflammatory bowel disease, ${ }^{20}$ the use of natural compounds for the prevention and treatment of inflammatory bowel disease shows an increasing trend.
Antioxidant, anti-inflammatory, antimicrobial, and wound healing properties of these compounds ${ }^{21}$ like aloe vera can be involved in the prevention and repair 


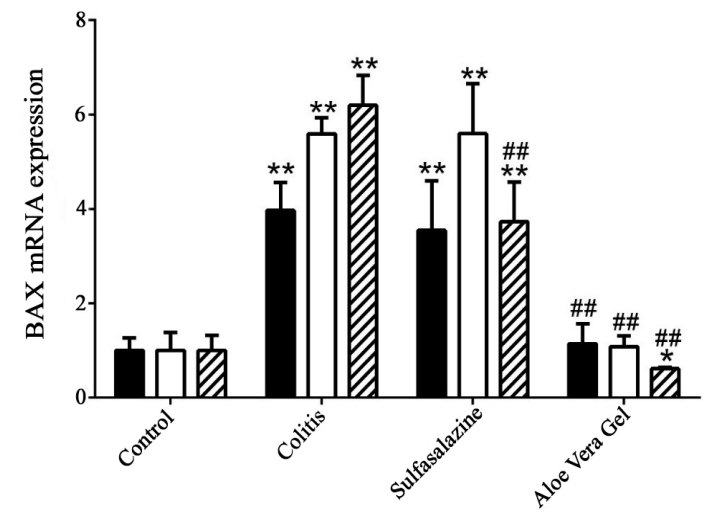

Chart 1: The expression of the Bax mRNA in different groups, over the 7-, 14-, and 21-day periods

of UC and even provide a protective effect on the creation of inflammation. ${ }^{10,11,12,14,15}$ The anti-inflammatory effect of aloe vera is associated with a decrease in the production of nitric oxide that leads to a decrease in the release of inflammatory mediators like TNF- $\alpha$ and an increase in anti-inflammatory mediators like IL-10. ${ }^{22,23}$ Also, aloe vera inhibited the production of PGE- IL-2 and IL- 8 responsible for inflammatory reactions. ${ }^{23,24}$ The antioxidant property of aloe vera is due to presence of various vitamins and minerals in this plant including vitamin $\mathrm{A}, \mathrm{E}, \mathrm{C}$, and selenium. ${ }^{25}$ The increase in activity of antioxidant enzymes, can strongly clear free radicals, like $\alpha$-tocopherol that finally leads to a reduction in inflammation and can accelerate healing and recovery. ${ }^{26,27}$

The glucomannan ingredient in aloe vera, which is a polysaccharide rich in mannose, and gibberellin can have interaction with several receptors and increase proliferation of fibroblasts and increase synthesis of collagen and proteoglycans and consequently accelerate wound healing. The glycoproteins which are present in aloe vera can prevent swelling and pain and accelerate the recovery process. ${ }^{12,14,17}$ Aloe vera not only increases the amount of collagen in the site of injury, but also changes the structure of collagen strands, and increases the connections between these strands and consequently accelerates wound healing. ${ }^{17} \beta$-sitosterol is another compound found in aloe vera that can increase the expression of vasculoendothelial growth factor (VEGF) leading to an increase in angiogenesis and a better healing in injured tissue. ${ }^{28}$

Considering several studies conducted in treatment of UC using alternative medicine and natural prod-

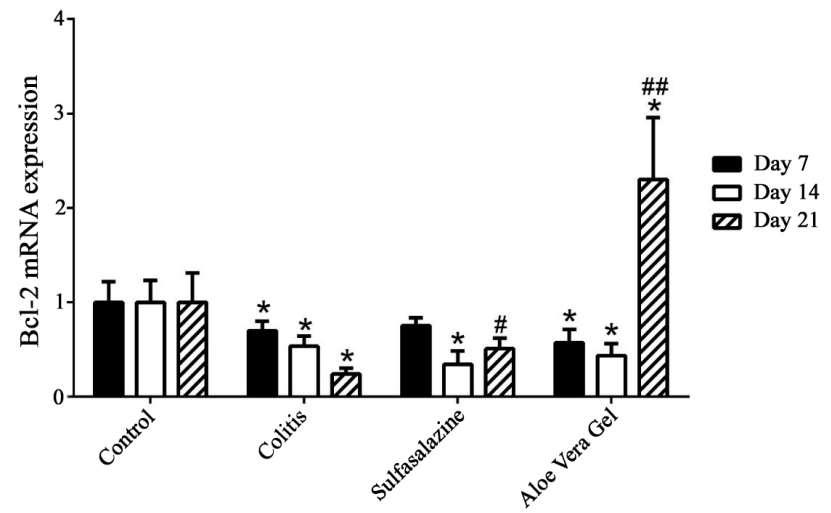

Chart 2: The expression of the Bcl-2 mRNA in different groups, over the 7-, 14-, and 21-day periods

ucts, ${ }^{2,5,6,8,9,12,18}$ the current study evaluated the effect of oral aloe vera gel in amelioration of acetic acid-induced colitis in an experimental model of rat by histopathological and molecular assessment methods. It was shown that aloe vera gel could significantly improve the histopathological indexes by reducing inflammation, edema, necrosis in the injured tissue. This improvement can be due to antioxidant and anti-inflammatory, antimicrobial, and wound healing properties of aloe vera that was mentioned earlier. $^{22,23,24,25,28,29}$

Dehury and his colleagues in 2017 investigated the protective effect of the aqueous leaf extract of aloe vera in an animal model of experimentally induced colitis observing improvement in histopathological indices in the treated group revaeling that the aqueous leaf extract of aloe vera could have a protective effect in UC. ${ }^{29}$ Our findings are in line with the study performed by Keshavarzi and his colleagues in 2014 assessing the effect of aqueous extract of aloe vera in gastric acid secretion and colonic histopathology in experimental UC model induced by acetic acid in rats. They demonstrated that the treatment with aloe vera could reduce the severity of histopathological symptoms. ${ }^{30}$ In a recent study by increasing the length of treatment and using more accurate molecular evaluation methods, opposite outcomes were found. In Wiener et al.'s study, the researchers emphasized the use of sulfasalazine to cause worsening of injuries in experimental colitis due to an increase in tissue reactions. ${ }^{31}$ Topcu-Tarladacalisir and colleagues demonstrated that curcumin had beneficial effects on colitis induced by acetic acid in rats by decreasing the inflammatory responses and 
levels of oxidative stress and apoptotic cell death. ${ }^{32}$

We showed in the $21^{\text {st }}$ day that apoptosis was significantly less when compared with the colitis group left without treatment. Aloe vera in the $21^{\text {st }}$ day was demonstrated to decrease apoptosis and accelerate healing process in the intestinal tissue due to a significant reduction in Bax mRNA expression and a significant elevation in Bcl-2 mRNA expression when compared with colitis group without treatment. It seems that the reduction of apoptosis can be due to the antioxidant and anti-inflammatory effects of aloe vera proved in previous studies. ${ }^{22,23,24,25,28,29}$

\section{CONCLUSION}

Our study highlighted the evidence for the healing effects of aloe vera gel on acetic acid-induced experimental model of UC. These favorable effects were determined with the improvement of histopathological indices in colonic tissue and reduction in apoptosis by downregulation of Bax gene and upregulation of Bcl-2 anti-apoptotic gene. These findings can be added to the literature when targeting aloe vera in treatment of UC.

\section{ACKNOWLEDGMENT}

Thanks to Shiraz University of Medical Sciences for financial support of this study.

\section{ETHICAL APPROVAL}

There is nothing to be declared.

\section{CONFLICT OF INTEREST}

The authors declare no conflict of interest related to this work.

\section{REFERENCES}

1. Safarpour AR, Hosseini SV, Mehrabani D. Epidemiology of inflammatory bowel diseases in iran and Asia; a mini review. Iran J Med Sci 2013;38:140-9.

2. Mehrabani D, Bahrami F, Hosseini SV, Ashraf MJ, Tanideh N, Rezaianzadeh A, et al. The healing effect of teucrium polium in acetic acid induced ulcerative colitis in the dog as an animal model. Middle East $J$ Dig Dis 2012;4:41-8.

3. Shamsdin SA, Saberifiroozi M, Mehrabani D, Heydari ST. Pepsinogen I and II, gastrin and cag A serum levels in Shiraz. Middle East J Dig Dis 2011;3:103-9.

4. Podolsky DK. Inflammatory bowel disease. $N$ Engl $J$ Med 2002;347:417-29. doi: 10.1056/NEJMra020831.
5. Hosseinzadeh F, Salehi M, Tanideh N, Sepehrimanesh M, Mehrabani D, Sayarifard A, et al. Investigation of the enema administration of grape seed oil with or without sesame oil on acetic acid induced ulcerative colitis in rats. World J Plast Surg 2017;6:176-82.

6. Khodakarm-Tafti A, Mehrabani D, Homafar L, Farjanikish G. Healing effects of Elaeagnus angustifolia extract in experimentally induced ulcerative colitis in rats. $J$ Pharmacol Toxicol 2015;10:29-35.

7. Honardoost M, Soleimanjahi H, Rajaei F. Apoptosis: programmed cell death. Review Article. JQUMS 2013;17:48-57.

8. Takhshid MA, Mehrabani D, Ai J, Zarepor M. The healing effect of licorice extract in acetic acid-induced ulcerative colitis in rat model. Comp Clin Pathol 2012;21:1139-44. doi: 10.1007/s00580-011-1249-9.

9. Mehrabani D, Ziaei M, Hosseini SV, Ghahramani L, Bananzadeh AM, Ashraf MJ, et al. The effect of calendula officinalis in therapy of acetic acid induced ulcerative colitis in dog as an animal model. Iran Red Crescent Med J 2011;13:884-90.

10. Kaser A, Zeissig S, Blumberg RS. Inflammatory bowel disease. Annu Rev Immunol 2010;28:573-621. doi: 10.1146/annurev-immunol-030409-101225.

11. Podolsky DK. Inflammatory bowel disease. $N$ Engl $J$ Med 2002;347:417-29. doi: 10.1056/NEJMra020831.

12. Tanideh N, Nematollahi S, Hosseini SV, Hosseinzadeh M, Mehrabani D, Safarpour A, et al. The healing effect of hydroalcoholic extract of hypericum perforatum on acetic acid-induced ulcerative colitis in male rats. $J$ Fasa Univ Med Sci 2016;6:530-7.

13. Ogura Y, Bonen DK, Inohara N, Nicolae DL, Chen FF, Ramos $\mathrm{R}$, et al. A frame shift mutation in NOD2 associated with susceptibility to Crohn's disease. Nature 2001;411:6036. doi: $10.1038 / 35079114$.

14. Ordás I1, Eckmann L, Talamini M, Baumgart DC, Sandborn WJ. Ulcerative colitis Lancet 2012;380:1606-19. doi: 10.1016/S0140-6736(12)60150-0.

15. Grucela A, Steinhagen RM. Current surgical management of ulcerative colitis. Mt Sinai J Med 2009;76:606-12. doi: $10.1002 / \mathrm{msj} .20152$.

16. Goldstein N, Dulai M. Contemporary morphologic definition of backwash ileitis in ulcerative colitis and features that distinguish it from crohn disease. $\mathrm{Am} \mathrm{J}$ Clin Pathol 2006;126:365-76. doi: 10.1309/UAXMW3428PGN9HJ3.

17. Arab HH, Al-Shorbagy MY, Abdallah DM, Nassar NN. Telmisartan Attenuates Colon Inflammation, Oxidative Perturbations and Apoptosis in a Rat Model of Experimental Inflammatory Bowel Disease. PLOS ONE 2014;9:e97193. doi:10.1371/journal.pone.0097193. 
18. Tanideh N, Bahrani M, Khoshnood-Mansoorkhani MJ, Mehrabani D, A Firoozi D, Koohi-Hosseinabadi $\mathrm{O}$, et al. Evaluating the effect of melillotus officinalis 1. aqueous extracts on healing of acetic acid-induced ulcerative colitis in male rats. Ann Colorectal Res 2016;4:e42856. doi: 10.17795/acr-42856.

19. Al-Rejaie SS, Abuohashish HM, Al-Enazi MM, AlAssaf AH, Parmar MY, Ahmed MM. Protective effect of naringenin on acetic acid-induced ulcerative colitis in rats. World $J$ Gastroenterol 2013;19:5633-44. doi: 10.3748/wjg.v19.i34.5633.

20. Mehrabani D, Vahedi M, Eftekhari MH, Amini M, Masoumi SJ. Food Avoidance in patients with ulcerative colitis. Int J Nutr Sci 2017;2:189-95.

21. Hassanshahi N, Masoumi SJ. The effect of omega-3 fatty acids in ulcerative colitis. Int J Nutr Sci 2018;3:58-64.

22. Sholehvar F, Mehraban D, Yaghmaei P, Vahdati A. The effect of aloe vera gel on viability of dental pulp stem cells. Dent Traumatol 2016;32:390-6. doi: 10.1111/ edt.12272.

23. Langmead L, Feakins M, Goldthorpe S, Holt H, Tsironi E, Silva AD, et al. Randomized, double-blind, placebo-controlled trial of oral Aloe Vera gel for active ulcerative colitis. Aliment Pharmacol Ther 2004;19: 739-47. doi: 10.1111/j.1365-2036.2004.01902.x

24. Eamlamnam K, Patumraj S, Visedopas N, Thong-Ngam D. Effects of Aloe Vera and sucralfate on gastric microcirculatory changes, cytokine levels and gastric ulcer healing in rats. World J Gastroenterol 2006;12:2034-9. doi: 10.3748/wjg.v12.i13.2034.

25. Hajhashemi V, Ghannadi A, Heidari AH. Antiinflammatory and wound healing activities of Aloe littoralis in rats. Res Pharm Sci 2012;7:73-8.

26. Pierce GF, Vande Berg J, Rudolph R, Tarpley J, Mustoe TA. Platelet-drived growth factor BB and transforming growth factor-beta 1selectivly modulate glycosaminoglycans, collagen, and myofibroblasts in ecisional wounds. Am J Pathol 1991;138:629-46.

27. Coskun S, Gulec EG, Balabanli B, Acarturk F. Effects of epidermal growth factor on lipid peroxidation and nitric oxide levels in oral mucosal ulcer healing. A time-course study. Surg Today 2007;37:570-4. doi: 10.1007/s00595-006-3455-7.

28. Moon EJ, Lee YM, Lee OH, Lee MJ, Lee SK, Chung $\mathrm{MH}$, et al. A novel angiogenic factor derived from Aloe vera gel: $\beta$-sitosterol, a plant sterol. Angiogenesis 1999;3:117-23. doi: 10.1023/a:1009058232389.

29. Dehury S, Tripathy J. Effect of Aloe Vera Leaf Extract in Animal Model of Ulcerative colitis. Ejpmr 2017,4,346-50.

30. Keshavarzi Z, Alikhani V, Vatanchian M, Tabatabaei Yazdi A, Bibak B, Mohebbati R. Effects of Aloe Vera
Gel on Gastric Acid Secretion and Colon Histopathology in Ulcerative Colitis Model induced by Acetic Acid in Rats. J Zanjan Univ Med Sci Health Service 2014;22:1-11.

31. Wiener C, Fauci AS, Braunwald E, Kasper DL, Hauser SL, Longo DL, et al. Harrison's principles of internal medicine, self-assessment and board review: McGraw Hill Professional 2008.

32. Topcu-Tarladacalisir Y, Akpolat M, Hulya Uz, Kizilay G, Sapmaz-Metin M, Cerkezkayabekir A, et al. Effects of Curcumin on Apoptosis and Oxidoinflammatory Regulation in a Rat Model of Acetic Acid-Induced Colitis: The Roles of c-Jun N-Terminal Kinase and p38 Mitogen-Activated Protein Kinase. J Med Food 2013;16,296-305. doi: 10.1089/jmf.2012.2550. 